\title{
From Concept to Crystals via Prediction: Multi-Component Organic Cage Pots by Social Self-Sorting
}

\author{
Rebecca L. Greenaway, ${ }^{[a]}$ Valentina Santolini, ${ }^{[b]}$ Angeles Pulido, ${ }^{[c, d]}$ Marc A. Little, ${ }^{[a]}$ Ben M. Alston, ${ }^{[a]}$ \\ Michael. E. Briggs, ${ }^{[\mathrm{a}]}$ Graeme M. Day, ${ }^{[\mathrm{c}]^{*}}$ Andrew I. Cooper ${ }^{[\mathrm{a}]^{*}}$ and Kim E. Jelfs ${ }^{[\mathrm{b}]^{*}}$
}

\begin{abstract}
We describe the a priori computational prediction and realization of multi-component cage pots, starting with molecular predictions based on candidate precursors through to crystal structure prediction and synthesis using robotic screening. The molecules were formed by the social self-sorting of a tri-topic aldehyde with both a tritopic amine and di-topic amine, without using orthogonal reactivity or precursors of the same topicity. Crystal structure prediction suggested a rich polymorphic landscape, where there was an overall preference for chiral recognition to form heterochiral rather than homochiral packings, with heterochiral pairs being more likely to pack window-towindow to form two-component capsules. These crystal packing preferences were then observed in experimental crystal structures.
\end{abstract}

\section{Introduction}

Porous organic cages (POCs) are discrete self-assembled molecules that contain a permanent intrinsic cavity. They are typically formed from a binary mixture of precursors using dynamic covalent chemistries. ${ }^{[1-4]}$ POCs lend themselves to crystal engineering approaches, ${ }^{[5,6]}$ and their crystal packing can be directed to form porous solids. ${ }^{[7]}$ Porous cages have been used in a range of applications including gas adsorption and molecular separations. ${ }^{[8,9]}$ The a priori design of these molecules remains challenging, however, both in terms of synthesis and crystal engineering. In the synthesis step, different potential cage topologies may be possible, and it can be hard to predict the synthetic outcome based on the precursors. It is also difficult to

[a] Dr R. L. Greenaway, Dr M. A. Little, Dr B. M. Alston, Dr M. E. Briggs, Prof. A. I. Cooper FRS

Department of Chemistry and Materials Innovation Factory University of Liverpool

51 Oxford Street, Liverpool, L7 3NY, UK.

Email: aicooper@liverpool.ac.uk

[b] Dr V. Santolini, Dr K. E. Jelfs

Department of Chemistry

Imperial College London

Molecular Sciences Research Hub, White City Campus, Wood

Lane, London W12 OBZ, UK.

Email:k.jelfs@imperial.ac.uk

[c] Dr A. Pulido, Prof. G. M. Day

School of Chemistry

University of Southampton

Highfield, Southampton, SO17 1BJ, UK.

Email:G.M.Day@soton.ac.uk

[d] Dr A. Pulido

Current address: The Cambridge Crystallographic Data Centre, 12 Union Road, Cambridge, CB2 1EZ, UK.

Supporting information for this article is given via a link at the end of the document. anticipate how the resulting cages will pack together in a crystal. This combined uncertainty in both the molecular synthesis step and the crystallization step makes it essentially impossible to design solid-state function from knowledge of the cage precursors alone. The use of more than two distinct cage precursors and the number of possible outcomes makes this even more challenging. For example, with a ternary mixture of precursors, one can observe narcissistic self-sorting into separate binary cages, ${ }^{[10-12]}$ social self-sorting into a mixed assembly, ${ }^{[13,14]}$ 'scrambling' to form statistical mixtures of products, ${ }^{[15-18]}$ or polymer formation. Hence, developing methods to predict the synthetic outcome for combinations of more than two precursors could greatly enhance our ability to access organic materials with new properties, particularly if we can couple this with methods to predict solidstate crystallization. Our vision, therefore, is an in silico 'precursors-to-crystals' design paradigm that can deal with complex multi-component supramolecular assemblies.

To date, there are just a few reports of the social self-sorting of more than two distinct components into discrete cage species, most likely because of the difficulty in designing these specific, multi-component assemblies. ${ }^{[19]}$ Recently, Klotzbach and Beuerle demonstrated that the condensation between a tri-topic catechol linker and a mixture of di-topic boronic acids can lead to either the discrete formation of separate two-component cages (narcissistic self-sorting), or the formation of a three-component cage (social self-sorting), depending on the combination of 'bite-angles' present on the di-topic linkers. ${ }^{[13]}$ Mastalerz and co-workers also demonstrated the ability to favor the formation of a socially selfsorted organic cage using a mixture of tri-topic amines by

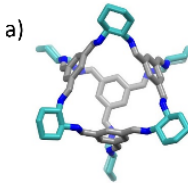

cC3

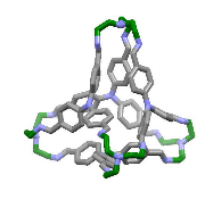

CC11

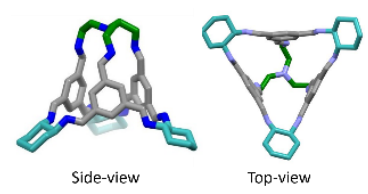

OCP3
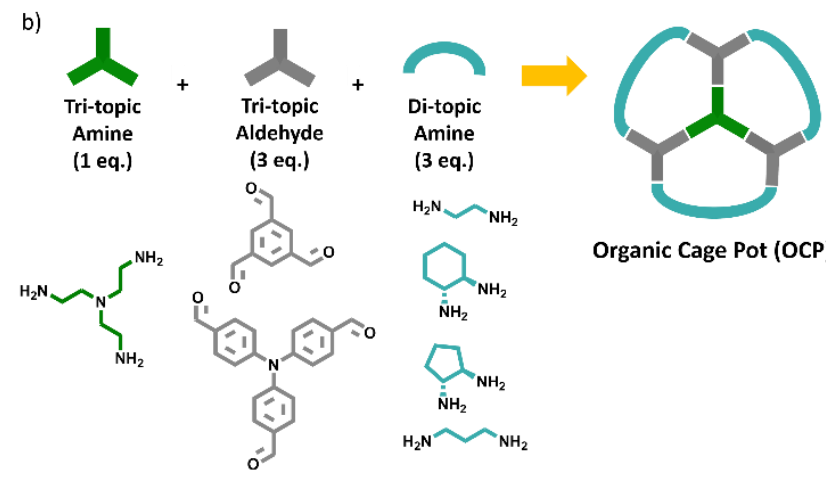

Organic Cage Pot (OCP)

Figure 1. (a) Design inspiration for multi-component cage pots - the combination of a cage window, such as that found in CC3, with the capping triamine used in CC11 was used to target ternary organic cage 'pots' such as OCP3; (b) Representation of the organic cage pots and their constituent parts, along with the precursors selected for screening. 
exploiting differences in solubility compared to the narcissistically self-sorted 'parent' cages. ${ }^{[14]}$ Cooper and co-workers showed that the condensation between a tri-topic aldehyde and a mixture of di-topic vicinal diamines led to neither social nor narcissistic selfsorting, but instead formed a statistical distribution of 'scrambled' cages with mixed vertices. ${ }^{[15]}$ An alternative strategy that can also lead to successful social self-sorting is to integrate orthogonal reactivity: for example, Severin and co-workers reported the clean formation of a three-component organic cage using mechanochemistry by employing both imine and boronic acid condensations. ${ }^{[20]}$

The self-sorting of multi-component cages is typically approached by mixing precursors in a dynamic combinatorial style, and (sometimes) post-rationalized using computational modelling. There is now an increasing drive to use computational prediction prior to synthesis as a tool to guide the discovery of useful and increasingly complex species. This is valuable because the synthesis and characterization of a new molecule can often take many months in the laboratory. In recent years, advances in computational modelling have enabled the reliable design and prediction of the formation of organic cages, including the topology most likely to be formed by synthesis ${ }^{[21-23]}$ and the subsequent crystal structure packing, including preferences for forming racemic or enantiopure forms. ${ }^{[6,24-26]}$ However, a priori computational design becomes increasingly complicated when the number of distinct components increases because of the potential to form different self-assembled competing products.

Our aim here was to predict and then synthesize a capping unit, or 'cage pot', that incorporates a cage window ${ }^{[27,28]}$ for chiral recognition with related imine cages (Figure 1a) and a chiral binding site. ${ }^{[29,30]}$ We successfully predicted and then realized three-component organic cage pots (OCPs) formed via the social self-sorting of a tri-topic amine, di-topic amine and tri-topic aldehyde (Figure 1a). The formation of these cage pots does not require orthogonal reactivity, and it does not use an additional component of the same topicity; that is, it does not use two tritopic or di-topic amines with a tri-topic aldehyde, as exploited in other examples. ${ }^{[13,14,19,20]}$ Crystal structure prediction (CSP) was carried out a priori on the cage pots that contained a 'CC3like ${ }^{\text {'[31][32] }}$ window as the common assembly motif, allowing in silico screening to determine the lowest energy crystal structures, and their potential for porosity, chiral recognition between cages, and polymorphism. Previously, the self-sorting of two precursors into a specific cage topology has been predicted, as has the crystal packing of known, two-component cages..$^{[6,21,24,33]}$ However, this study is the first example of the design and prediction for POCs all the way from the cage precursors to CSP, coupled with experimental robotic screening to realize the predicted products.

\section{Results and Discussion}

With a cage pot topology in mind as the design target, we selected a range of precursors where the di-topic amine and tri-topic aldehyde were varied while using the same tri-topic amine throughout, thus giving five different hypothetical cage pots (Figure 1b). Models of each 'pot' were built and analyzed prior to any laboratory work (Figure 2). For each cage pot, the low-energy

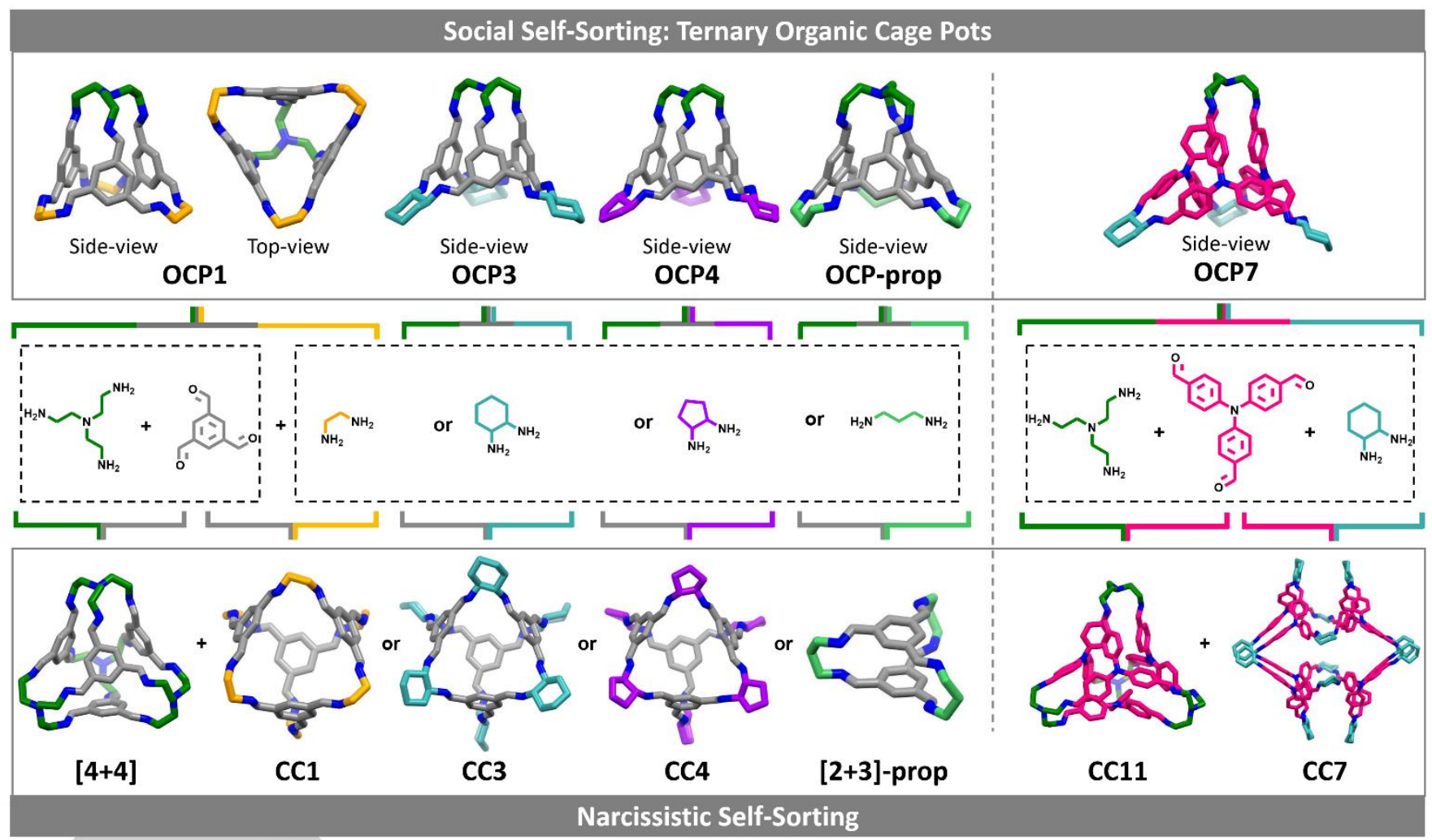

Figure 2 Computational models of the hypothetical organic cage pots (OCPs) that could be formed if social self-sorting occurs using a tri-topic amine, tri-topic aldehyde, and di-topic amine (top), and the complementary binary parent cages that could form if narcissistic self-sorting occurs (bottom). Hydrogens are omitted for clarity. The number assigned to each OCP relates to the binary parent cage that is usually formed from the reaction between the tri-topic aldehyde and the di-topic amine, for example, OCP1 relates to CC1, OCP3 relates to CC3, OCP7 relates to CC7, etc. 
conformations were explored with molecular dynamics using the OPLS3 force field, ${ }^{[34]}$ before using density functional theory (DFT) calculations to optimize those structures for a more accurate energetic ranking at the PBE/TZVP-MOLOPT level in CP2K with a Grimme-D3 dispersion correction. For full computational details, refer to section 1 of the supporting information. To allow direct comparison of the formation energies with the 'parent' cages that could be formed if narcissistic self-sorting occurred between the trialdehyde with either the di-topic or tri-topic amines, the energies were normalized by calculating the formation energy per imine bond formed (Table 1). ${ }^{[23]}$ While this approach does not consider effects such as solvation or the kinetic route to the cages, these formation energies have been shown previously to correlate with experimental trends for cage viability, in particular ruling out cases with unfavorable formation energies. ${ }^{[23]}$ Generally, the cage pot formation energies were similar to the parent cage molecules, suggesting that the socially self-sorted pots were synthetically viable, with the formation energy per imine bond for OCP3 (-19 kJ $\left.\mathrm{mol}^{-1}\right)$ and OCP7 $\left(-20 \mathrm{~kJ} \mathrm{~mol}^{-1}\right)$ being the most energetically favorable.

Table 1. Comparison of the formation energies per imine bond formed for the three-component socially self-sorted cage pots, with the two-component narcissistically self-sorted parent cages.

\begin{tabular}{|c|c|c|c|}
\hline \multicolumn{4}{|c|}{ Assembly } \\
\hline $\begin{array}{l}\text { Socially } \\
\text { Self-Sorted }\end{array}$ & $\begin{array}{l}\text { Formation Energy } \\
\text { per Imine Bond } \\
\text { Formed }\left(\mathrm{kJ} \mathrm{mol}^{-1}\right)\end{array}$ & $\begin{array}{l}\text { Narcissistically } \\
\text { Self-Sorted }\end{array}$ & $\begin{array}{l}\text { Formation Energy } \\
\text { per Imine Bond } \\
\text { Formed }\left(\mathrm{kJ} \mathrm{mol}^{-1}\right)\end{array}$ \\
\hline OCP1 & -13.7 & $\begin{array}{l}{[4+4]} \\
\text { CC1 }\end{array}$ & $\begin{array}{l}-20.6 \\
-13.5\end{array}$ \\
\hline OCP3 & -19.2 & $\begin{array}{l}{[4+4]} \\
\text { cc3 }\end{array}$ & $\begin{array}{l}-20.6 \\
-21.3\end{array}$ \\
\hline OCP4 & -15.7 & $\begin{array}{l}{[4+4]} \\
\text { CC4 }\end{array}$ & $\begin{array}{l}-20.6 \\
-18.5\end{array}$ \\
\hline OCP-prop & -14.4 & $\begin{array}{c}{[4+4]} \\
{[2+3]-\text { prop }}\end{array}$ & $\begin{array}{l}-20.6 \\
-10.2\end{array}$ \\
\hline OCP7 & -19.6 & CC7 & -21.6 \\
\hline
\end{tabular}

We next carried out a high-throughput synthetic screen of the different multi-component cage pots, alongside the twocomponent parent cages as controls (Figure 2). Overall, this led to five reactions containing all three components (OCPs: tri-topic amine, tri-topic aldehyde, and di-topic amine), and seven reactions containing two components (one of: tri-topic amine and tri-topic aldehyde, or tri-topic aldehyde and di-topic amine). For full details of the high-throughput synthesis methods, refer to section 3 of the supporting information. Analysis of the multicomponent reaction mixtures using ${ }^{1} \mathrm{H}$ NMR spectroscopy and high-resolution mass spectrometry (HRMS) suggested the successful formation of two organic cage pots-OCP3 and OCP7
- the same two assemblies that were predicted a priori to be the most energetically favorable. Further analysis suggested that the major products were the socially self-sorted cage pots, with a small amount of narcissistic self-sorting to the binary cages CC3 and CC11, respectively, which tallies with the formation energies that were predicted to be similar to the multi-component cage pots. CC3 and OCP3 could be separated by analytical HPLC using our standard optimized conditions for organic cages (Figure S9), confirming a ratio of $\sim 2: 1$ OCP3:CC3 that corresponded with that observed in the ${ }^{1} \mathrm{H}$ NMR spectra (Figure S11), suggesting that purification by preparative-HPLC was possible. However, CC11 and OCP7 had very similar retention times (Figure S12), which meant that OCP7 could not be isolated by preparative-HPLC, although the ${ }^{1} \mathrm{H}$ NMR spectra suggested a favorable $~ 8: 1$ ratio of OCP7:CC11 had been formed (Figure S14). With a successful hit for the formation of an organic cage pot, and the ability to purify it by preparative-HPLC, OCP3 was investigated further; the synthesis was scaled up, and the use of different enantiomers of the di-topic amine $(R, R ; S, S$; rac) were investigated to afford OCP3- $R$, OCP3-S, and OCP3-rac respectively, for use in crystallization studies.

During this process, the reactions were monitored at room and elevated temperature, and whilst the formation of the pot was apparent after 2 days, after prolonged heating for 5 days, the reaction had reached equilibrium with no further change apparent in the product mixture in the spectra, and the majority of sideproducts had been converted to either OCP3 or CC3 (Figure S15S17). Further, the solution of equilibrated species was found to be stable on standing. However, the ratio of OCP3:CC3 was found to improve, from $\sim 2: 1$ in the high-throughput screen, to between $\sim 5.5: 1$ and $\sim 13: 1$ (see section 4 of the supporting information). This suggests that the ratio of pot to cage formed experimentally is directly influenced by the ratio of precursors used, with accurate quantities of each precursor required to favor and achieve the highest conversion to the socially self-sorted cage pots, which is possible with less error during scale-up.

The results pose an interesting question; is our observed formation of OCP3 and OCP7 via social self-sorting thermodynamically or kinetically driven? While experimentally the ratio of self-sorting to narcissistic-sorting ranged from $\sim 5.5: 1$ to $\sim 13: 1$, the DFT calculated formation energies suggest that the energetic driving force favours narcissistic self-sorting over cage pot formation, albeit by a small margin, especially given potential DFT errors of a few $\mathrm{kJ} \mathrm{mol}^{-1}$. However, these calculations do not account for entropy differences, which should favour the social self-sorting for both OCP3 and OCP7 on the basis of a smaller loss of rigid molecule (rotational and translational) entropy; this is due to the larger number of product molecules for social sorting. An estimate of entropic contributions to the free energy difference is provided in section 1.1 of the supporting information, which supports the thermodynamic argument: the entropic advantage outweighs the formation energy difference for OCP3 and OCP7, but not for OCP4 and OCP-prop, while energy and entropy are finely balanced for OCP1. Further, we neglect the influence of solvent, which could influence the relative energies of the reaction outcomes, for instance the contribution of some non-covalent interactions in stabilizing the molecules may be exaggerated in gas-phase calculations. 

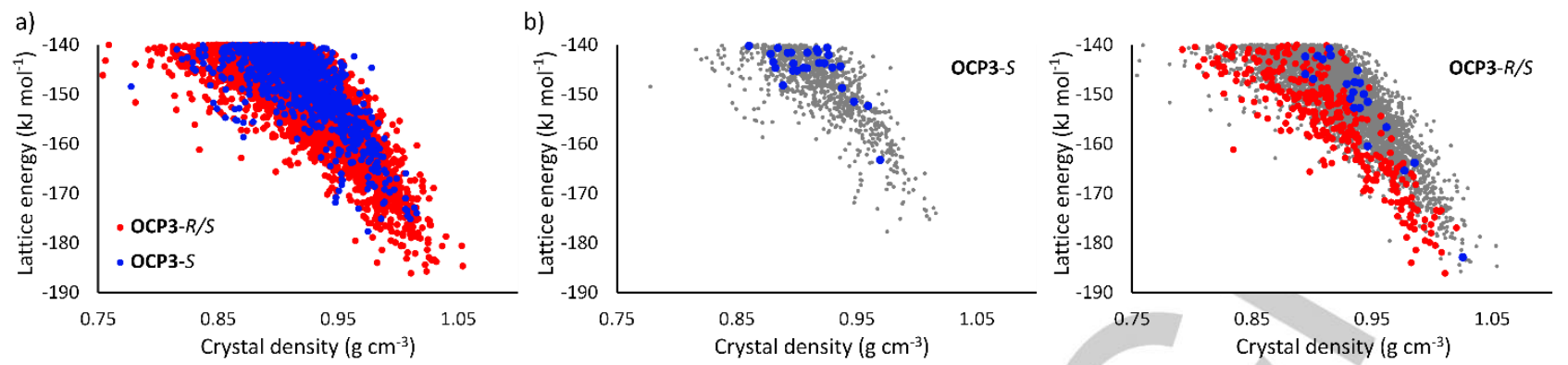

c)
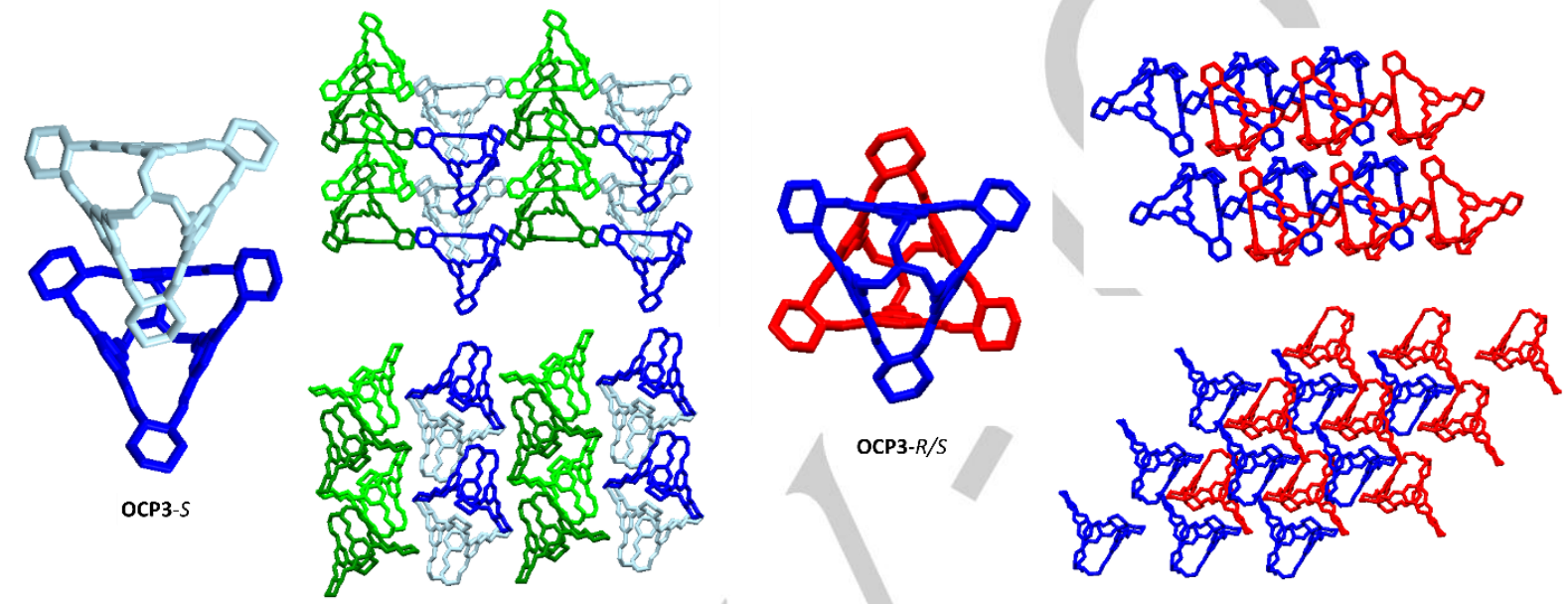

Figure 3 (a) Lattice energy rankings for OCP3, with racemate structures (OCP3- $R / S$ ) shown in red and enantiopure structures (OCP3-S) shown in blue; (b) Windowto-window packing preferences over the CSP energy landscapes for homochiral OCP3-S and racemate OCP3- $R / S$ : blue data points are structures with homochiral window-to-window interactions, red data points are structures with heterochiral window-to-window interactions; (c) Lowest energy predicted homochiral OCP3-S structure, showing a staggered vertex-to-window packing, and racemic OCP3- $R / S$, showing window-to-window packing

In some systems, solvent choice is known to influence the reaction outcome, and it was estimated that the free energy

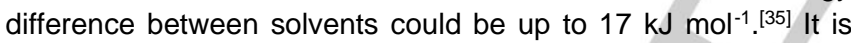
therefore plausible that differences in the relative solvent stabilization between the reactants and products could influence the experimentally observed product ratios. It is also known that kinetic trapping of cage products can occur, ${ }^{[36]}$ which we did not consider here as it is extremely computationally demanding to get accurate free energies for every step of the reaction pathway. Given the uncertainties in entropic and solvent contributions, we cannot be certain whether kinetic or thermodynamic effects drive the preference for self-sorting in this case; this remains an open question for much of supramolecular chemistry.

Before investigating the crystallization of these organic cage pots, CSP was carried out to evaluate the lattice energies for candidate crystal packings, and to investigate the potential of these cage pots for porosity, chiral recognition, and polymorphism in the solid state. Prior to CSP, we studied the window-to-window interaction between the pots using dispersion-corrected DFT (DFT-D) cluster models of the dimers, to compare the strength of the interaction to that in $\mathbf{C C} 3$ dimers which contain the same windows. It was found that the strength of the window-to-window interaction is only slightly weaker for OCP3 $\left(-171 \mathrm{~kJ} \mathrm{~mol}^{-1}\right)$ when compared to CC3 $\left(-198 \mathrm{~kJ} \mathrm{~mol}^{-1}\right)$. Alongside this, the dimer models also suggested that chiral recognition between the supramolecular cage species is preserved, with a stronger stabilization observed for the OCP3-R/OCP3-S dimer (-171 kJ $\left.\mathrm{mol}^{-1}\right)$ compared to OCP3-S/OCP3-S dimer $\left(-125 \mathrm{~kJ} \mathrm{~mol}^{-1}\right)$ based on the interaction energies (Table S2).

We next used CSP to obtain energy landscapes of predicted structures to provide information about the packing preferences of the pots. The predicted crystal structures for homochiral OCP3$S$ and racemic OCP3- $R / S$ show the typical trend of lattice energy decreasing with increasing density (Figure 3a), and the absence of any large energy gaps or low energy 'spikes' ${ }^{[37]}$ in the distribution of structures suggests that there is not one mode of crystal packing that is dominant. The results show that chiral recognition is generally preferred; OCP3- $R / S$ results in lower energy structures than homochiral OCP3-S, and the global minimum on the $R / S$ landscape is $8.4 \mathrm{~kJ} \mathrm{~mol}^{-1}$ more stable than the global minimum on the $S / S$ landscape. The preference for forming a racemate over an enantiopure system is similar to the observation for the parent cage $\mathbf{C C} \mathbf{3}_{{ }^{[24]}}$ but the energetic preference is larger (ca. $\left.30 \mathrm{~kJ} \mathrm{~mol}^{-1}\right)^{[6]}$ for $\mathbf{C C} 3$ because the chirality affects four window-to-window interactions per CC3, compared to only one window-to-window interaction per OCP3. Alongside this, when analyzing the packing preferences across the entire landscape, we find that the racemic OCP3-R/S structures are more likely to exhibit window-to-window packing than the homochiral OCP3-S/S structures (Figure $3 b$ ). Many of the predicted structures on the OCP3-R/S landscape exhibit window-to-window interactions, including the lowest energy structure and most structures that lie along the low energy edge of the landscape. 
a)

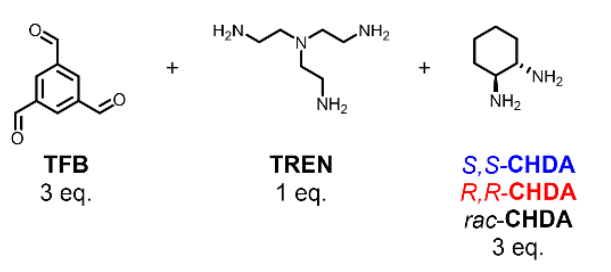

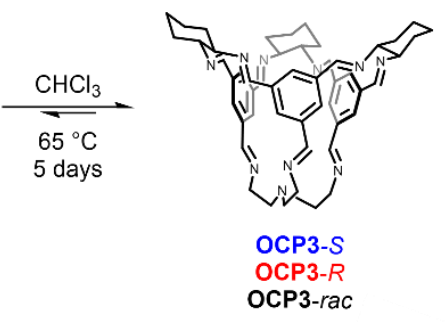

b)

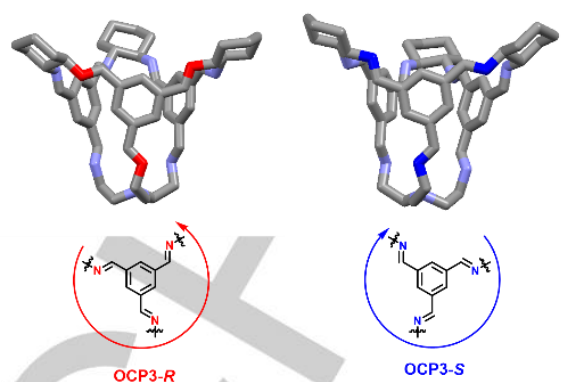

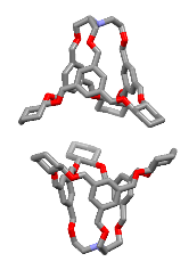

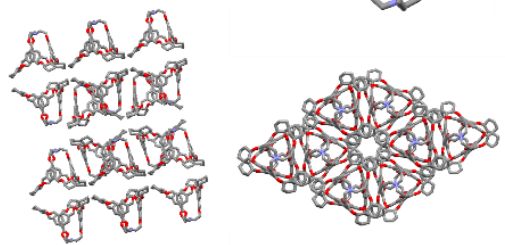

OCP3-R $\left(\mathrm{PG}_{3} 22\right)$

window-to-window
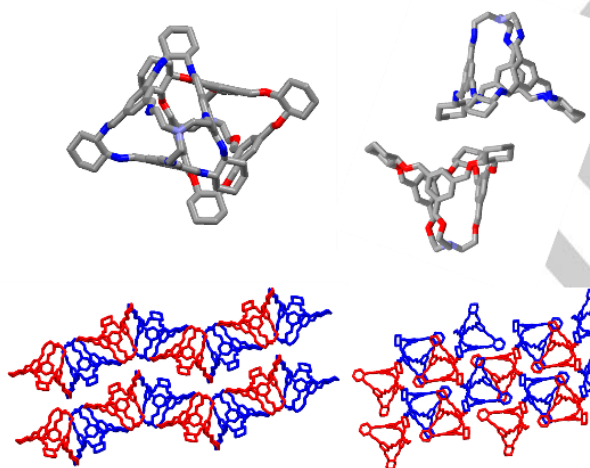

OCP3-R + OCP3-S (P2 1 /C)

Staggered $R / S$ window-to-window
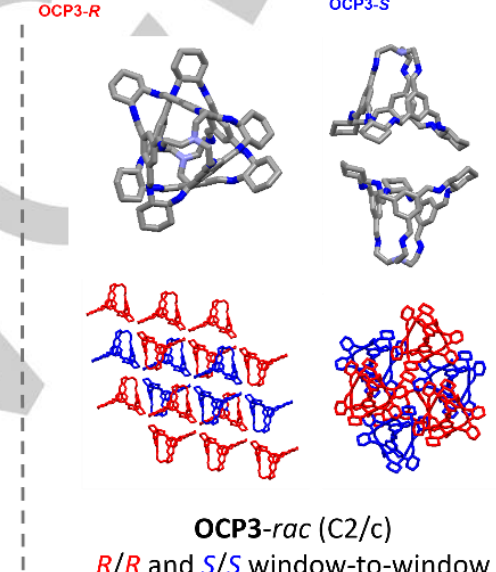

OCP3-rac (C2/c)

$R / R$ and $S / S$ window-to-window

Figure 4 (a) Synthesis of the self-sorted cage pot OCP3, formed from the reaction of a tri-topic aldehyde with both di-topic and tri-topic amines; (b) Homochiral organic cage pots are formed depending on the chirality of the vicinal diamine used: $S, S-C H D A$ leads to OCP3-S, and $R, R$-CHDA leads to OCP3-R; (c) Crystal structures grown from solutions of OCP3- $R$, a 1:1 mixture of homochiral OCP3- $R$ with homochiral OCP3-S, and OCP3-rac (formed directly using rac-CHDA in the multi-component one-pot reaction) in methanol - OCP3- $R$ are shown in red, OCP3-S are shown in blue, and hydrogens are omitted for clarity.

While both heterochiral (OCP3-R/S) and homochiral (separate OCP3-S/S and OCP3- $R / R$ pairs) window-to-window interactions are possible in OCP3-R/S structures, only a small number of the predicted structures contain homochiral window-to-window interactions (Figure 3b).

Despite the greater prevalence of heterochiral pairings on the OCP3-R/S CSP landscape, we find that the lowest energy structure with homochiral pairings is energetically competitive with the best structures with heterochiral pairs. Thus, crystal packing can override the inherent energetic preference for heterochiral interactions seen in the isolated dimer calculations. In contrast to OCP3-R/S, only a small proportion of OCP3-S predicted crystal structures contain window-to-window arrangements of OCP3 molecules and these are found in the higher energy regions of the landscape.

This preference can be seen clearly in the lowest energy predicted structures (Figure 3c), with the racemate OCP3- $R / S$ demonstrating window-to-window packing, whilst the homochiral OCP3-S/S shows a staggered vertex-to-window structure - this difference in preferential packing for the homo- and hetero-chiral crystals has also been observed previously in the TCC1-3 cage family. However, in both cases, there is a dense collection of lowenergy structures; as such, it can be expected that the systems might be polymorphic - for example, within $7.2 \mathrm{~kJ} \mathrm{~mol}^{-1}$ of the lowest energy structure, which is the typical energetic range of polymorphism, ${ }^{[38]}$ there are 14 structures for homochiral (OCP3$S / S$ ), and 27 for racemic cage pots (OCP3-R/S).

Overall, CSP suggested that for OCP3, chiral recognition can be expected but, with no single preferential crystal packing motif apparent, we expected these systems to have a high chance of being polymorphic. As such, solvent might play a key role in determining which structures are obtained. Solvent templating effects in cage systems have been found previously to have a large energetic influence; for example, in the case of $\mathbf{C C 1}$, different solvents stabilize structures over a range of approximately $50 \mathrm{~kJ} \mathrm{~mol}^{-1}$ on the computer crystal structure landscape ${ }^{[39]}$ Void analysis of the hypothetical landscapes for the OCP3 structures suggested that almost all of the low energy structures had closed, zero-dimensional pores, with a few lower density structures having 1-dimensional pores (Figure S1). The lowest energy structure from the CSP for OCP3-S has only isolated voids that are extrinsic to the pot (Figure S3), with a pore limiting diameter (PLD) of $2.8 \AA$ and a largest cavity diameter (LCD) of $4.6 \AA$, whereas OCP3-R/S has isolated voids with a mixture of intrinsic voids (between window-to-window packed pots) and extrinsic isolated voids (Figure S4), a PLD of $1.2 \AA$ and a LCD of $4.5 \AA$. These LCDs would be sufficient to host small gases, such as xenon and krypton.

While CSP suggested that the majority of structures would not possess any 1-, 2-, or 3-dimensional pore structures, as might be expected for these cage pots that possess only a single guest accessible window, we were still interested in confirming their crystal structures by experiment to test the CSP conclusions. To do this, analytically pure samples of each of OCP3- $R,-S$, and rac, were obtained using preparative-HPLC, and a crystallization screen was carried out. Single-crystals were grown by slow evaporation of methanol from homochiral OCP3- $R$, a 1:1 mixture of homochiral OCP3- $R$ with homochiral OCP3-S, and OCP3-rac, 
formed directly using rac-CHDA in the multi-component one-pot reaction (Figure 4).

Single crystal X-ray diffraction (SCXRD) confirmed that the molecular structure of the organic cage pot matched that which was predicted a priori using computational modelling, further confirming that the use of rac-CHDA led to narcissistically and socially self-sorted homochiral cage pots, OCP3- $R$ and OCP3- $S$. That is, the $R, R$-isomer in the racemic mixture formed OCP3- $R$, whilst the $S, S$-isomer separately formed OCP3-S, over the formation of CC3- $R$ and $\mathbf{C C 3}-S$ respectively.

All of the obtained crystal structures contained solvent molecules, both methanol and water, the presence of which precludes a direct comparison to the CSP landscape, which only considered pure OCP3 structures. However, several of the overall predictions made by CSP were confirmed. Crystallization from the 1:1 mixture of OCP3- $R$ and OCP3-S, as well as from OCP3-rac, led to racemic crystal structures, indicating that there is an energetic stability of OCP3- $R / S$ relative to a conglomerate of enantiomerically pure crystals of OCP3-S and OCP3- $R$. Solvatomorphism occurred in the racemates with different structures crystallising from 1:1 OCP3-R:OCP3-S $\left(P 2_{1} / C\right)$ and OCP3-rac (C2/c), both grown in methanol. Interestingly, the two racemic crystal structures contain different dimer pairs when packing window-to-window - 1:1 OCP3-R:OCP3-S showed heterochiral $R / S$ packing, while OCP3-rac showed homochiral $R / R$ and $S / S$ packing. This observation is in line with the small energetic difference between the lowest energy predicted structures with heterochiral and homochiral interactions on the OCP3-R/S CSP landscape. Finally, a preference for window-towindow packing was observed in all three experimental crystal structures, with the presence of $\mathrm{MeOH}$ frustrating the window-towindow arrangement. The window-to-window packing in OCP3- $R$ disagrees with the predictions from CSP; in this case, the energetic influence of solvent within the crystal structure has overridden the predicted packing preference of pure OCP3- $R$. Solvent inclusion in crystal structures of organic cages has been shown to provide significant stabilization, preferentially stabilizing cage packings up to about $60 \mathrm{~kJ} \mathrm{~mol}^{-1}$ above the most stable pure cage crystal structure. ${ }^{[39]}$ From our CSP results, this energy range includes window-to-window packing for enantiomerically pure OCP3 (Figure 3b). The result shows the importance of further developing methods to efficiently account for solvent during CSP.

The addition of a cage pot, OCP3, to our family of cage molecules with a CC3-type window, adds a new unit to our molecular cage toolkit. We hypothesized that OCP3 could provide a molecular "capping" unit when connected to other porous cages within the family via window-to-window interactions. To test this, we conducted a limited CSP study (see Supporting Information Section 1.4) to investigate the energy landscape of a $4: 1$ OCP3:CC3 co-crystal, to see whether packings with a cage pot capping each of the four windows of a CC3 unit was an energetically feasible packing arrangement. However, these calculations indicated that the 4:1 OCP3:CC3 packed poorly and the best predicted co-crystal was found to be over $100 \mathrm{~kJ} \mathrm{~mol}^{-1}$ (per 4:1 OCP3:CC3) less stable than the separate OPC3 and CC3 crystals (Figure S7). For this reason, co-crystallization was not explored experimentally.

\section{Conclusion}

In conclusion, we have successfully realized a new socially self-sorted supramolecular species-organic cage pots-that are formed from three distinct components without the exploitation of orthogonal reactivities to direct the assembly. A priori computational prediction was used to investigate the formation energies of the socially self-sorted organic cage pots, compared to the separate binary organic cages that would be obtained if narcissistic self-sorting occurred, and indicated that the new assemblies were synthetically viable. After synthetic realization in a high-throughput screen, a priori crystal structure prediction was used to investigate the crystal packing and potential properties of an organic cage pot. Solvent-free crystals were not obtained by experimental, preventing a direct comparison with the CSP, but this study highlights how a hybrid workflow fusing computation with experiment can guide the targeted discovery of new, complex supramolecular materials. ${ }^{[23]}$ These chiral organic cage pots have analogies to hemicryptophanes, ${ }^{[40]}$ which suggests that they might be of interest for the formation of guest-host complexes with the potential to encapsulate guests (e.g., rare gases, such as xenon)..$^{[41,42]}$

\section{Acknowledgements}

We acknowledge funding from the European Research Council under FP7 (RobOT, ERC Grant Agreement No. 321156, ANGLE, ERC Grant No. 307358, and CoMMaD, ERC Grant No. 758370), and the Engineering Research Council and Physical Sciences Research Council (EPSRC) (EP/M017257/1, EP/P005543/1, EP/N004884/1), including the UK's HEC Materials Chemistry Consortium (EP/L000202/1) for time on the UK supercomputer, ARCHER. We acknowledge the use of the IRIDIS High Performance Computing Facility, and associated support services at the University of Southampton. K.E.J. thanks the Royal Society for a University Research Fellowship. We thank the MicroBioRefinery for assistance with QTOF-MS measurements. We thank Dr. Filip Szczypiński for useful discussions.

\section{Conflict of Interest}

The authors declare no conflict of interest.

Keywords: crystal engineering $\cdot$ crystal structure prediction • molecular design $\bullet$ porous organic cages $\bullet$ self-sorting

\footnotetext{
[1] G. Zhang, M. Mastalerz, Chem. Soc. Rev. 2014, 43, 1934-1947.

[2] J. D. Evans, C. J. Sumby, C. J. Doonan, Chem. Lett. 2015, 44, 582 588 .

[3] T. Hasell, A. I. Cooper, Nat. Rev. Mater. 2016, 1, 16053.

[4] F. Beuerle, B. Gole, Angew. Chemie - Int. Ed. 2018, 57, 4850-4878.

[5] M. A. Little, M. E. Briggs, J. T. A. Jones, M. Schmidtmann, T. Hasell, S. Y. Chong, K. E. Jelfs, L. Chen, A. I. Cooper, Nat. Chem. 2015, 7, 153-159.

[6] J. T. A Jones, T. Hasell, X. Wu, J. Bacsa, K. E. Jelfs, M. Schmidtmann, S. Y. Chong, D. J. Adams, A. Trewin, F. Schiffman, et al., Nature 2011, 474, 367-371.

T. Hasell, J. L. Culshaw, S. Y. Chong, M. Schmidtmann, M. A. Little,
} 
K. E. Jelfs, E. O. Pyzer-Knapp, H. Shepherd, D. J. Adams, G. M. Day, et al., J. Am. Chem. Soc. 2014, 136, 1438-1448.

T. Mitra, K. E. Jelfs, M. Schmidtmann, A. Ahmed, S. Y. Chong, D. J. Adams, A. I. Cooper, Nat. Chem. 2013, 5, 276-281.

[9] L. Chen, P. S. Reiss, S. Y. Chong, D. Holden, K. E. Jelfs, T. Hasell, M. A. Little, A. Kewley, M. E. Briggs, A. Stephenson, et al., Nat. Mater. 2014, 13, 954-960.

[10] K. Acharyya, S. Mukherjee, P. S. Mukherjee, J. Am. Chem. Soc 2013, 135, 554-557.

[11] X. Wang, P. Peng, W. Xuan, Y. Wang, Y. Zhuang, Z. Tian, X. Cao, Org. Biomol. Chem. 2017, 16, 34-37.

[12] M. Kołodziejski, A. R. Stefankiewicz, J.-M. Lehn, Chem. Sci. 2019 DOI 10.1039/C8SC04598D.

[13] S. Klotzbach, F. Beuerle, Angew. Chemie - Int. Ed. 2015, 54, 10356-10360.

[14] D. Beaudoin, F. Rominger, M. Mastalerz, Angew. Chemie - Int. Ed. 2017, 56, 1244-1248.

[15] S. Jiang, J. T. A. Jones, T. Hasell, C. E. Blythe, D. J. Adams, A. Trewin, A. I. Cooper, Nat. Commun. 2011, 2, 207.

[16] Q. Wang, C. Zhang, B. C. Noll, H. Long, Y. Jin, W. Zhang, Angew. Chemie - Int. Ed. 2014, 53, 10663-10667.

[17] S. Lee, A. Yang, T. P. Moneypenny, J. S. Moore, J. Am. Chem. Soc. 2016, 138, 2182-2185.

[18] J. C. Lauer, W. S. Zhang, F. Rominger, R. R. Schröder, M. Mastalerz, Chem. - A Eur. J. 2018, 24, 1816-1820.

[19] K. Ono, N. Iwasawa, Chem. - A Eur. J. 2018, 24, 17856-17868.

[20] D. Tan, T. Friščić, European J. Org. Chem. 2018, 2018, 18-33.

[21] V. Santolini, M. Miklitz, E. Berardo, K. E. Jelfs, Nanoscale 2017, 5280-5298.

G. Zhu, Y. Liu, L. Flores, Z. R. Lee, C. W. Jones, D. A. Dixon, D. S. Sholl, R. P. Lively, Chem. Mater. 2017, 30, 262-272.

[23] R. L. Greenaway, V. Santolini, M. J. Bennison, B. M. Alston, C. Stackhouse, M. A. Little, M. Miklitz, E. G. B. Eden, R. Clowes, A. Shakil, et al., Nat. Commun. 2018, 9, 2849 E. O. Pyzer-Knapp, H. P. G. Thompson, F. Schiffmann, K. E. Jelfs, S. Y. Chong, M. A. Little, A. I. Cooper, G. M. Day, Chem. Sci. 2014, 5, 2235-2245.

M. Bernabei, R. Pérez-Soto, I. Gómez García, M. Haranczyk, Mol. Syst. Des. Eng. 2018, 3, 942-950.

[26] M. Bernabei, R. Pérez Soto, I. Gómez García, M. Haranczyk, Mol. Syst. Des. Eng. 2019, DOI 10.1039/c9me00018f.
Tozawa, J. T. A. Jones, S. I. Swamy, S. Jiang, D. J. Adams, S. Shakespeare, R. Clowes, D. Bradshaw, T. Hasell, S. Y. Chong, et al., Nat. Mater. 2009, 8, 973-978

[28] A. G. Slater, M. A. Little, A. Pulido, S. Y. Chong, D. Holden, L. Chen, C. Morgan, X. Wu, G. Cheng, R. Clowes, et al., Nat. Chem. 2016, 9, 17.

[29] M. E. Briggs, K. E. Jelfs, S. Y. Chong, C. Lester, M. Schmidtmann, D. J. Adams, A. I. Cooper, Cryst. Growth Des. 2013, 13, 4993-5000.

[30] T. Hasell, S. Y. Chong, K. E. Jelfs, D. J. Adams, A. I. Cooper, J. Am Chem. Soc. 2012, 134, 588-598.

[31] T. Tozawa, J. T. A. Jones, S. I. Swamy, S. Jiang, D. J. Adams, S. Shakespeare, R. Clowes, D. Bradshaw, T. Hasell, S. Y. Chong, et al., Nat. Mater. 2009, 8, 973.

[32] A. G. Slater, M. A. Little, A. Pulido, S. Y. Chong, D. Holden, L. Chen, C. Morgan, X. Wu, G. Cheng, R. Clowes, et al., Nat. Chem. 2017, 9, 17-25.

[33] A. G. Slater, P. S. Reiss, A. Pulido, M. A. Little, D. L. Holden, L. Chen, S. Y. Chong, B. M. Alston, R. Clowes, M. Haranczyk, et al., ACS Cent. Sci. 2017, 3, 734-742.

[34] E. Harder, W. Damm, J. Maple, C. Wu, M. Reboul, J. Y. Xiang, L. Wang, D. Lupyan, M. K. Dahlgren, J. L. Knight, et al., J. Chem. Theory Comput. 2016, 12, 281-296.

[35] X. Liu, R. Warmuth, J. Am. Chem. Soc. 2006, 128, 14120-14127.

[36] T. P. Moneypenny, A. Yang, N. P. Walter, T. J. Woods, D. L. Gray, Y. Zhang, J. S. Moore, J. Am. Chem. Soc. 2018, 140, 5825-5833.

[37] A. Pulido, L. Chen, T. Kaczorowski, D. Holden, M. A. Little, S. Y. Chong, B. J. Slater, D. P. McMahon, B. Bonillo, C. J. Stackhouse, et al. Nature 2017, 543, 657-664.

[38] J. Nyman, G. M. Day, CrystEngComm 2015, 17, 5154-5165

[39] D. P. McMahon, A. Stephenson, S. Y. Chong, M. A. Little, J. T. A. Jones, A. I. Cooper, G. M. Day, Faraday Discuss. 2018, 211, 383399.

[40] D. Zhang, A. Martinez, J. P. Dutasta, Chem. Rev. 2017, 117, 49004942 .

[41] C. M. Kane, O. Ugono, L. J. Barbour, K. T. Holman, Chem. Mater 2015, 27, 7337-7354

. T. T. Holman, J. Am. Chem. Soc. 2016, 138, 4377-4392.

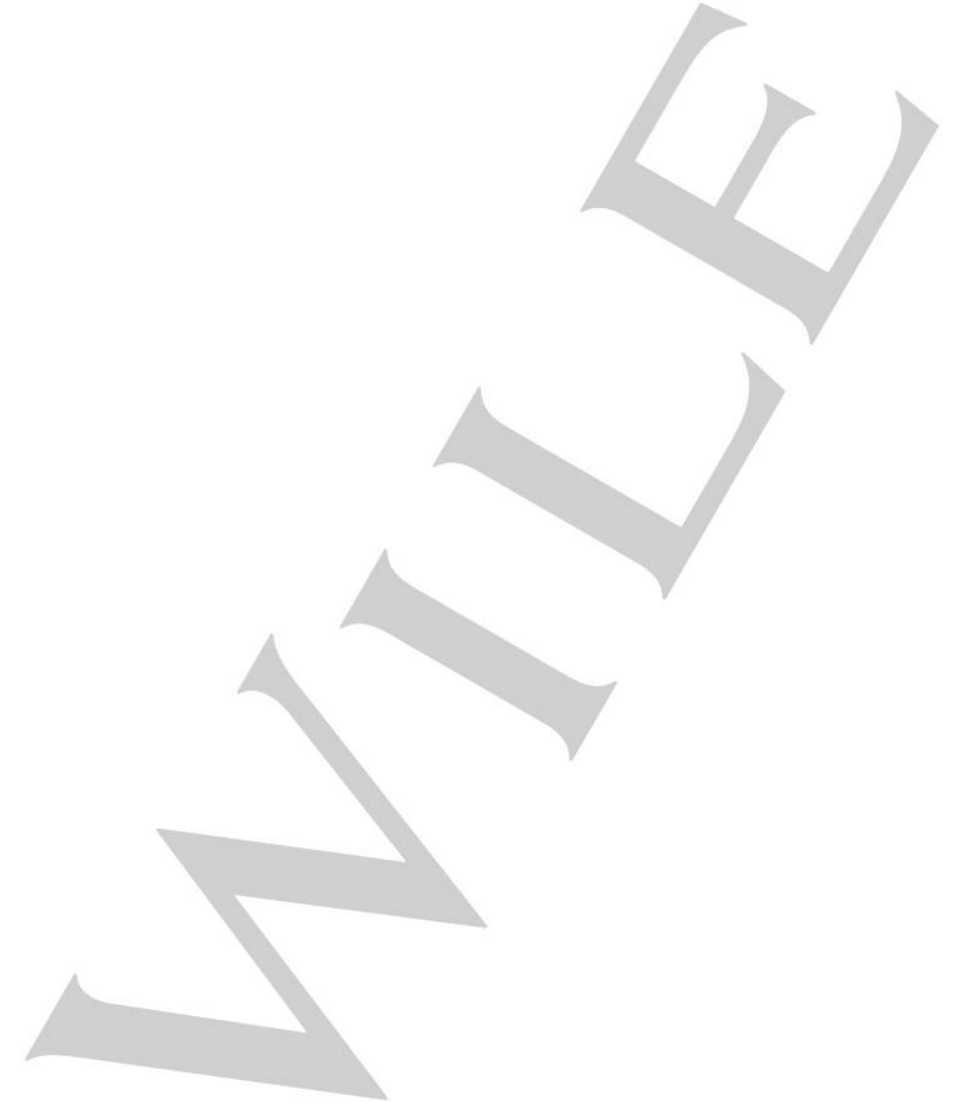




\section{Entry for the Table of Contents}

\section{RESEARCH ARTICLE}

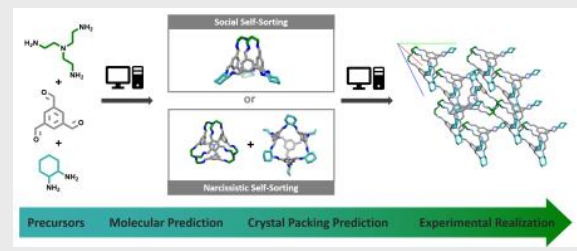

From molecular structure to solid-state assembly, we first computationally predict and then synthetically realize a multi-component self-sorted organic cage pot
Rebecca L. Greenaway, ${ }^{[a]}$ Valentina Santolini, ${ }^{[b]}$ Angeles Pulido, ${ }^{[c]}$ Marc A. Little, ${ }^{[a]}$ Ben M. Alston, ${ }^{[a]}$ Michael. E. Briggs, ${ }^{[a]}$ Graeme M. Day, ${ }^{[c]^{*}}$ Andrew I. Cooper ${ }^{[a]^{*}}$ and Kim E. Jelfs ${ }^{[b]^{*}}$

Page No. - Page No.

From Concept to Crystals via Prediction: Multi-Component Organic Cage Pots by Social Self-Sorting 